\section{Cahiers de Narratologie}

Analyse et théorie narratives

12 | 2005

Récit et éthique

\title{
L'inscription du code de valeurs féodal dans la construction narrative de Vie de Saint-Louis
}

Ilias Yocaris

\section{(2) OpenEdition}

1 Journals

\section{Édition électronique}

URL : http://journals.openedition.org/narratologie/30

DOI : 10.4000/narratologie.30

ISSN : 1765-307X

Éditeur

LIRCES

\section{Référence électronique}

Ilias Yocaris, "L'inscription du code de valeurs féodal dans la construction narrative de Vie de SaintLouis », Cahiers de Narratologie [En ligne], 12 | 2005, mis en ligne le 20 avril 2005, consulté le 15 novembre 2019. URL : http://journals.openedition.org/narratologie/30 ; DOI : 10.4000/narratologie.30

Ce document a été généré automatiquement le 15 novembre 2019.

\section{(c) (i) (9)}

Cahiers de Narratologie - Analyse et théorie narratives est mis à disposition selon les termes de la licence Creative Commons Attribution - Pas d'Utilisation Commerciale - Pas de Modification 4.0 International. 


\title{
L'inscription du code de valeurs féodal dans la construction narrative de Vie de Saint-Louis
}

\author{
Ilias Yocaris
}

1 Écrite en 1309, la Vie de Saint-Louis constitue une sorte d'hagiographie, ou plutôt de biographie pieuse : l'auteur, noble champenois de haut rang, évoque les hauts faits de Louis IX (1226-1270), et notamment la croisade que ce dernier a entreprise en Égypte contre les Sarrasins (1248-1254). Deux choses frappent le lecteur contemporain dans la construction narrative de Vie de Saint-Louis: 1. La manière dont le récit de Joinville réfléchit la vision du monde et le code de valeurs féodaux au niveau de ses invariants structurels et stylistiques; 2 . Corrélativement, la manière dont cette inscription du code de valeurs féodal dans la construction narrative du texte entre en conflit avec sa logique hagiographique.

2 Comment le code de valeurs féodal se trouve-t-il inscrit dans la construction narrative $\mathrm{du}$ texte de Joinville? Le maitre mot pour résumer la vision du monde et le code de valeurs féodaux est « continuité ».

3 Tout d'abord, il y a continuité absolue de chaque individu avec les autres au sein du tissu social, que ce soit à travers les rapports de lignage, de suzeraineté/vassalité ou encore de voisinage spatial (appartenance à la même région). Au niveau thématique, Joinville insiste de façon très visible sur tous ces rapports. Ainsi par exemple, dans le $\S$ 95 du texte, on met en avant de manière laudative la décision de Louis IX de faire la paix avec le roi d'Angleterre parce que leurs femmes sont sœurs et leurs enfants cousins germains. Cette continuité du tissu social féodal trouve son équivalent isomorphe dans la construction du récit par l'emploi très fréquent d'un procédé stylistique récurrent, très cinématographique : la présentation, dans les descriptions, $\mathrm{du}$ roi, des grands seigneurs et de Joinville par une espèce de "travelling " métonymique qui va de l'un à l'autre et les montre toujours comme un groupe continu qui «fait bloc ». Exemples : la description, au § 93, d'un repas de Louis IX à Saumur. Joinville, qui a alors 16 ans, assiste à ce repas en tant qu'écuyer tranchant de Thibaut de 
Champagne. Nous traduisons : «À la table du roi mangeait, auprès de lui, le comte de Poitiers [...] ; et après le comte de Poitiers mangeait le comte Jean de Dreux [...] ; après le comte de Dreux mangeait le comte de la Marche [...] ; après le comte de la Marche le bon comte Pierre de Bretagne [...] ; et devant la table du roi, en face du comte de Dreux, mangeait messire le roi de Navarre [...] ; et je tranchais la viande devant lui. ». Ici, la progression métonymique de la description vise de toute évidence à suggérer deux choses: (a) d'une part, comme on l'a déjà souligné, que le roi forme avec les nobles de son entourage un tout homogène qui «fait bloc "; (b) d'autre part, comme on le verra en détail plus loin, qu'il existe une sorte de continuité entre le roi et Joinville, et même une sorte d'équivalence de leurs fonctions respectives, la phrase s'ouvrant par l'évocation du roi et se terminant par celle de Joinville. Cet effet du travelling métonymique est tout à fait conscient, d'où sa reprise par exemple au $\$ 108$ (où l'on passe successivement en revue les principaux croisés, en commençant par le roi et en terminant encore par Joinville) ou encore au $\$ 378$ (nous traduisons) : « Ils jetèrent une planche à terre pour embarquer le roi et le comte d'Anjou son frère et messire Geoffroy de Sargines et monseigneur Philippe de Nemours, et le maréchal de France que l'on appelait du Mez et le maître de la Trinité et moi ». Ici évidemment l'idée de la continuité entre les membres de la haute noblesse et/ou entre Joinville et le roi est suggérée à la fois par la progression métonymique du texte et par l'emploi du polysyndète.

4 Ensuite, il y a continuité entre royauté et christianisme, d'où, au niveau du récit, la présence de symétries et de détails descriptionnels qui permettent de croiser de façon persistante les mots « roi » et «croix »: Louis IX est né le jour de Saint-Marc, où l'on porte en procession des « Croiz noires » (\$69); c'est encore le jour de la Saint-Marc qu'il quitte Acre pour revenir en France à la fin de sa croisade, et le Narrateur lui dit qu'il se trouve « renez » de ce fait (dernière phrase du \$617). Or, dans la suite immédiate du texte (première phrase du \$618), l’on précise que le roi passe ensuite avec son bateau devant une montagne de Chypre «que en appelle la montaingne de la Croiz.». Bien entendu, le roi meurt aussi « en celle hore meismes que le Filz Dieu morut pour le salut du monde en la croiz» (\$757). On notera évidemment que le rapport d'assimilation implicite de Louis IX avec le Christ ainsi obtenu est surdéterminé par la paronomase $[\mathrm{rw \varepsilon}] /[\mathrm{krw \varepsilon}]$.

5 Enfin, dans le texte de Joinville, il y a continuité entre subjectif et objectif, entre «monde réel » et «représentation imaginaire » : la construction du récit joinvillien montre que ce dernier est la manifestation d'une vision du monde qui ne connaît pas encore le "partage cartésien » de la connaissance entre res cogitans et res extensa. Le récit met ainsi sur le même plan sans les différencier les descriptions de Louis tel que Joinville l'a vu "en réalité » avec ses apparitions dans les rêves de Joinville (v. p. ex. § 731, 766-767). On constate aussi qu'il n'y a aucune différenciation dans le texte entre le réel et le mythique. Ainsi par exemple Joinville insère dans son récit comme témoignage véridique le récit d'un écuyer sauvé de la noyade par Notre-Dame-deVauvert, au début du $\S 651$ (nous traduisons) : «Je lui demandai comment il se faisait qu'il n'avait pas cherché à se sauver, ni en nageant ni d'une autre manière. Il me répondit qu'il n'y avait ni utilité ni besoin de réagir, car, dès qu'il commença à tomber, il se recommanda à Notre-Dame-de-Vauvert; et celle-ci le soutint par les épaules dès qu'il tomba, jusqu'à ce que la galère du roi l'ait recueilli. En l'honneur de ce miracle, je l'ai fait peindre à Joinville dans ma chapelle, et sur les vitraux de Blécourt. » 
Cette notion de la continuité trouve sa représentation « visuelle » à travers une mise en abyme métatextuelle : l'image du tissu, omniprésente dans le texte de Joinville. Cette image métaphorise à la fois :

- la continuité générationnelle et sociale, comme on le voit dans l'épisode des § 35-36, où Joinville, piqué d'entendre Robert de Sorbon lui dire qu'il est « plus noblement vestu que le roy ", réplique que lui au moins a hérité le tissu luxueux de ses vêtements de son père et de sa mère, qui étaient nobles, alors que Robert de Sorbon, tout aussi luxueusement habillé, est « filz de vilain et de vilainne ».

- la continuité métonymique du texte narratif lui-même, comme le montre la fameuse expression « entrelacier nostre matiere » (\$ 280).

- la continuité entre la royauté et le christianisme, d'où l'épisode où l'on voit Louis IX faire cadeau aux émissaires Tartares qui se sont présentés à lui d'une chapelle en étoffe où il a fait broder tous les mystères de la foi chrétienne (\$ 471$)$.

Dans ce contexte, quel est le traitement réservé à la figure royale? L'intention de Joinville, bruyamment manifestée au niveau du contenu thématique du texte, est d'écrire une hagiographie, donc de mettre en avant la figure du roi Louis IX (dont il est le vassal) comme individualité qui se détache complètement de son environnement immédiat et de son contexte historique pour devenir un exemplum intemporel. Toutefois, l'étude de la construction du récit joinvillien montre que cette intentio auctoris entre manifestement en conflit avec la vision du monde qui est celle de Joinville en tant que sujet narrant qui fait partie de la noblesse féodale, et ce pour au moins deux raisons.

8 (a) Tout d'abord parce que le récit de Joinville répercute inévitablement la vision du roi et de la royauté qui est celle de son milieu social, la haute noblesse (et Dieu sait si Joinville est quelqu'un qui a une conscience de classe très développée ...). Or, la haute noblesse voit justement le roi non point comme une figure qui se détache de son environnement, mais à l'inverse comme un primus inter pares qui fait partie intégrante de la classe sociale des grands seigneurs, qui se situe justement dans la continuité par rapport à eux. D'où le traitement très ambigu réservé par Joinville à la singularité avérée de Louis IX, celle-ci étant traitée tantôt sur le mode de l'éloge et tantôt - de manière à peine voilée - sur le mode du blâme, ce qui est pour le moins étrange dans une hagiographie. Dans sa partie purement hagiographique (essentiellement les 68 premiers paragraphes), le récit se focalise souvent sur la figure du roi tout seul adoptant des postures et prodiguant des propos "didactiques", comme les saints de l'iconographie catholique ( $\$ 37, \S 59$ etc.) : la singularisation du roi est ici évidemment valorisée, et ce dernier fait plus que jamais figure d'exemple. Toutefois, de manière très significative, dès que commencent les récits des combats de la croisade, deux cas de figure se présentent.

Soit la singularité du roi par rapport aux autres représentants de la haute noblesse se trouve habilement gommée, ce dernier apparaissant comme un primus inter pares. Ainsi par exemple, dans le $\$ 228$, on voit le roi marcher au combat dépassant ses gens des épaules, mais sans en apparaître détaché. Nous traduisons: "Pendant que j'étais à pied [...], le roi arriva avec tout son corps de bataille, au milieu d'un grand bruit de trompes et de timbales; et il s'arrêta à un chemin sur une levée de terre. Jamais je ne vis un homme en armes aussi beau, car il paraissait être au-dessus de ses gens, les dépassant des épaules ». On voit bien ici comment le texte insiste sur l'« esprit de corps » féodal et 
la continuité absolue qui existe entre le roi et ses hommes, qui forment un bloc unitaire.

Soit au contraire cette singularité est mise en avant mais dans ce cas toujours de manière négative : dans le $\S 162$, par exemple, le roi, qui vient d'arriver en Orient, près de Damiette, est à peine retenu par ses gens pour ne pas partir tout seul à l'abordage contre les Sarrasins; dans le $\S 183$, Joinville insiste ouvertement sur le fait que la décision désastreuse d'aller assiéger Le Caire (ce qui a éloigné l'armée française de ses sources de ravitaillement) a été prise parce que Louis IX s'est désolidarisé de "ses barons", autrement dit les grands seigneurs de son entourage (qui lui conseillaient tous d'attaquer Alexandrie pour avoir des possibilités de support logistique) pour n'écouter que son frère, le comte d'Artois.

11 (b) Mais le projet hagiographique de Joinville entre aussi en contradiction avec sa position personnelle par rapport aux événements qu'il évoque dans son récit. Sur le plan individuel, il est évident que Joinville éprouve la fierté du noble de haut rang qui a accédé à de hautes fonctions auprès de son roi et s'est vu confier par ce dernier des missions importantes. Dès lors, il a fatalement tendance à se mettre en avant, à suggérer au lecteur qu'il a joué un rôle important dans le déroulement de la croisade, qu'il a été même le partenaire privilégié du roi. Et cette volonté du Sujet narrant de se mettre en avant entre inévitablement en conflit avec la dimension hagiographique du texte: dans Vie de Saint-Louis, Joinville, le sujet de l'énonciation, aurait dû être en termes genettiens un narrateur témoin, n'ayant d'autre fonction que de rapporter ce qu'il a pu voir et entendre concernant le parcours de Saint-Louis. Or, le trait structural le plus marqué du texte, c'est une tentative incessante de transformer ce narrateur témoin en narrateur héros, qui n'est plus rejeté à la périphérie mais occupe au contraire le centre du récit : on sent bien à la lecture du texte qu'on n'a pas affaire à l'humble moine-scripteur d'une hagiographie qui s'efface lui-même devant la figure du saint qu'il évoque mais à un grand seigneur qui est fier de son propre parcours et le suggère ouvertement au lecteur. Cette occupation du devant de la scène par Joinville atteint sans doute son point culminant dans les $\$ 307-308$, lors du récit de la fuite des croisés pour échapper aux Sarrasins après la campagne militaire désastreuse sur les bords du Nil. Dans ce passage, Joinville se focalise tellement sur sa propre histoire qu'il finit par évoquer les circonstances de la capture du roi lui-même par les Sarrasins dans une digression, ce qui est assurément un comble! Nous traduisons : «On nous criait, à nous qui naviguions au fil de l'eau, [les croisés s'enfuient sur leurs galères], d'attendre le roi ; et quand nous ne voulions pas l'attendre, on tirait sur nous des carreaux d'arbalète, ce qui nous obligeait à nous arrêter jusqu'à ce qu'on nous donne l'autorisation de naviguer. § Je cesserai maintenant de vous parler de ce sujet et je vous dirai comment le roi fut pris, ainsi que lui-même me le conta. ».

Cette tentative de Joinville de se transformer en narrateur-héros se manifeste concrètement de trois façons différentes.

13 - Tout d'abord, lors du récit de la croisade, Joinville cherche à se présenter comme un personnage qui influence de manière déterminante le cours des événements. On pense notamment à l'épisode du conseil d'Acre, narré aux $\$ 419-434$. Ce conseil a lieu une fois le roi et les nobles de son entourage libérés par les Sarrasins, qui les avaient capturés. On discute pour savoir s'il faut ou non poursuivre la croisade (ce qui eût été impensable par exemple sous Louis XIV, qui aurait convoqué tous les nobles... pour leur notifier sa décision), et tous les grands seigneurs demandent au roi de rentrer en France. Or, 
Joinville, qui est un des derniers à prendre la parole, s'oppose violemment à cette idée et soutient, seul contre tous, que tout le monde doit rester et poursuivre la croisade. Le roi décide de suivre son avis et de rester: le narrateur-témoin s'est transformé en narrateur-héros.

- Le récit suggère aussi de plus en plus ouvertement l'existence d'un rapport privilégié entre le roi et Joinville, rapport fondé sur l'exclusion progressive de tous les intermédiaires entre eux. Pour le montrer, il suffit d'étudier la disposition des scènes où Joinville se retrouve face au roi. Dans le texte on peut dénombrer 19 scènes de ce genre, et il est facile de montrer qu'elles se succèdent selon une progression très significative en quatre temps :

位 la leçon à Joinville, qui reste confiné dans un rôle d'auditeur/observateur plus ou moins passif (§ 26-28, 32, 37-38, 39-40).

Dans un deuxième temps, Joinville adopte une attitude beaucoup plus active auprès du roi : il lui enlève son heaume et lui donne son chapeau de fer (§ 243), il intervient auprès des templiers pour qu'ils complètent de leurs fonds ce qui manquait pour payer sa rançon (§ 381-385), il lui donne un conseil que le roi suivra contre l'avis de tous ses conseillers ( $\$ 419-434$ ), et enfin il entreprend une négociation financière avec lui ( $\$$ 439-440). On voit donc Joinville avoir un rôle de plus en plus important auprès du roi, jusqu'à devenir pratiquement son égal. Et ça va encore plus loin :

17 Dans un troisième temps, on voit Joinville faire successivement une demande au roi ( $\S$ 499-500), un reproche parce que ce dernier a rompu les « convenances » faites entre eux (§505-506), des plaintes ( $\$ 509-510)$ auxquelles le roi obtempère, puis même lui imposer sa volonté aux §589-590 et l'accuser deux fois, aux §594 et 603-604. Joinville se présente donc lui-même comme ayant pris un ascendant sur le roi.

Enfin, quatrième et dernière étape du processus, Joinville devient successivement le compagnon préféré du roi ( $\$ 649$ ), son premier conseiller ( $\$ 653$ ), celui qui devine ses pensées (§ 655-656), celui qui pressent son avenir (\$ 731-732) et finalement celui qui le revoit encore en rêve après sa mort (\$766-767). On voit ainsi l'intimité entre le roi et Joinville atteindre un tel degré qu'il y a d'abord exclusion de tout intermédiaire entre eux, puis intériorisation finale de la figure du roi, qui devient une simple projection mentale de Joinville.

19 - Enfin, Joinville cherche à se mettre lui-même en avant en se présentant d'une certaine façon comme le dépositaire de la figure et du parcours de Louis IX. Cette tentative d'appropriation de la figure royale par Joinville se trouve symboliquement exprimée au niveau de la construction narrative du texte de deux façons :

- Tout d'abord, par l'organisation même du récit de la vie de Louis IX, qui s'étend du $\$ 69$ au $\S$ 765. Joinville prend soin d'évoquer, au début de ce récit, un fait qui semble sans rapport avec la narration et surtout sans intérêt : au $§ 84$, on voit ainsi Simon de Joinville, père du narrateur, prendre le parti du roi contre les grands seigneurs qui sont en conflit avec lui et menacent la ville de Troyes, que Simon se charge de défendre. Ce paragraphe n'a strictement aucune fonction narrative, puisqu'il est dit qu'en définitive les nobles en conflit avec le roi passèrent devant Troyes sans attaquer la ville. Mais le non-événement évoqué là prend toute sa signification si on juxtapose ce paragraphe avec le $\S 762$, où l'on voit Henri de Villars, neveu de Joinville et archevêque de Lyon, porter avec d'autres personnes le cadavre de Louis IX. On comprend ainsi que si Joinville dispose ces deux épisodes de part et d'autre du récit de la période où il a côtoyé Louis, c'est pour prolonger et renforcer sa propre 
relation avec le roi, même sur un mode purement fantasmatique, les membres de son lignage fonctionnant si l'on veut comme des extensions métonymiques de sa propre personne : on retrouve encore cette notion de la continuité si caractéristique du code de valeurs féodal. La portée de cet effet de symétrie macrostructurale est évidemment d'autant plus grande qu'il n'est pas immédiatement repérable.

- Ensuite, par la tentative de Joinville de s'approprier les reliques de Louis IX afin de les mettre dans la chapelle Saint-Laurent qu'il a lui-même construite à l'honneur du roi-saint ( $\$$ 767). Il n'est pas difficile de comprendre que le vieux sénéchal tente ainsi de s'approprier symboliquement non pas seulement la figure du défunt roi « elle-même ", mais aussi le témoignage de son parcours dans sa totalité : rappelons que le mot « reliques » vient du latin reliquia, « restes ", d'où la possibilité d'un rapprochement sémique avec le mot « témoignage ", qui évoque ce qui reste derrière nous, ce qu'on lègue à la postérité. Il va de soi que ce souci très marqué de laisser un témoignage de son parcours à la postérité s'inscrit également dans le cadre d'une pensée et d'une vision du monde dominées par l'idée de la continuité.

Ce qui frappe donc en définitive le lecteur contemporain dans Vie de Saint-Louis, c'est la manière dont ce texte "fait sens » à tous les niveaux, dont sa construction narrative et stylistique surdétermine son contenu thématique. Le fait que les moindres détails de la narration et de la structure sont agencés (malgré l'apparente confusion du récit) en fonction d'une stratégie verbale bien précise montre sans doute à quel point Joinville avait bien intériorisé le mode de pensée et d'expression très " codifié » qui est celui de la noblesse féodale de son époque. Mais n'est-ce pas là ce qu'admiraient en lui ses contemporains?

\section{RÉSUMÉS}

Deux éléments attirent tout de suite l'attention dans la construction narrative de Vie de SaintLouis: (a) L'inscription de la vision du monde et du code de valeurs féodaux au niveau des invariants structurels et stylistiques du récit ; (b) Le conflit entre le code de valeurs féodal et la logique hagiographique du texte.

(a) La vision du monde féodale, telle qu'elle se dégage du texte, repose pour l'essentiel sur la notion de continuité: continuité entre individus au sein du tissu générationnel et social, continuité entre royauté et christianisme, continuité entre subjectif et objectif, mythe et histoire, « réel » et « imaginaire ». Or, cette continuité se trouve métaphoriquement « exprimée » (au sens goodmanien du terme) par une série de procédés narratifs et stylistiques qui relèvent de la structure même du récit joinvillien, et non plus de son contenu thématique «de surface »: on peut citer par exemple l'emploi systématique de polysyndètes et d'une progression du type métonymique dans les descriptions du roi et de son entourage, le croisement persistant des mots

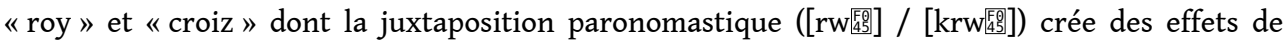
surdétermination assez visibles, ou encore le caractère récurrent dans le texte de l'image du tissu, qui constitue visiblement une mise en abyme métatextuelle.

(b) Toutefois, cette apologie implicite des valeurs féodales entre visiblement en conflit avec la logique hagiographique du récit joinvillien. Tout d'abord, dans la mesure où ce dernier doit 
mettre en avant la figure du roi-saint, il doit montrer comment cette figure se détache de son entourage, ce qui est incompatible avec la représentation féodale du roi comme primus inter pares et génère une série d'ambiguïtés et de contradictions au niveau de la "mise en scène " narrative du parcours de Louis IX. Ensuite, le narrateur, visiblement très fier d'appartenir à la haute noblesse et d'avoir été proche du roi, ne se contente nullement de la posture humble d'un narrateur-témoin (posture qui eût été propice à son travail hagiographique) et tente sans cesse de se transformer en narrateur-héros. Il en résulte un "décentrement » narratif du texte assez visible par moments, la structure du récit joinvillien suggérant ainsi que le narrateur n'entend point s'effacer derrière son roi, mais bien plutôt se mettre sur le même plan que lui.

INDEX

Mots-clés : style et contexte historique, vision du monde féodale, invariants narratifs et codification idéologique

Index chronologique : XIVe siècle

\section{AUTEUR}

\section{ILIAS YOCARIS}

Maître de conférences en littérature française à l'IUFM de Nice, membre du Centre Transdisciplinaire d'Épistémologie de la Littérature. Ilias Yocaris, IUFM de Nice 89, av. George V 06046 Nice Cedex 0493533386 y.ilias@wanadoo.fr 